\title{
Riluzole-Triazole Hybrids as Novel Chemical Probes for Neuroprotection in Amyotrophic Lateral Sclerosis
}

\author{
Joseph B. Sweeney, ${ }^{* \dagger}$ Marcus Rattray, ${ }^{*},+$ Victoria Pugh, ${ }^{\ddagger}$, and Lucy A. Powell ${ }^{\|}$ \\ ${ }^{\dagger}$ Department of Chemistry, Lancaster University, Lancaster LA1 4YB, U.K. \\ ${ }^{\ddagger}$ School of Pharmacy and Medical Sciences, University of Bradford, Bradford BD7 1DP, U.K. \\ ${ }^{\S}$ School of Chemistry, Food \& Nutritional Sciences and Pharmacy, University of Reading, Reading, Berkshire RG6 6AP, U.K. \\ "Department of Chemical Sciences, University of Huddersfield, Huddersfield HD1 3DH, U.K.
}

\section{Supporting Information}

\begin{abstract}
Despite intense attention from biomedical and chemical researchers, there are few approved treatments for amyotrophic lateral sclerosis (ALS), with only riluzole (Rilutek) and edaravone (Radicava) currently available to patients. Moreover, the mechanistic basis of the activity of these drugs is currently not well-defined, limiting the ability to design new medicines for ALS. This Letter describes the synthesis of triazole-containing riluzole analogues, and their testing in a novel neuroprotective assay. Seven compounds were identified as having neuroprotective activity, with two compounds having similar activity to riluzole.
\end{abstract}

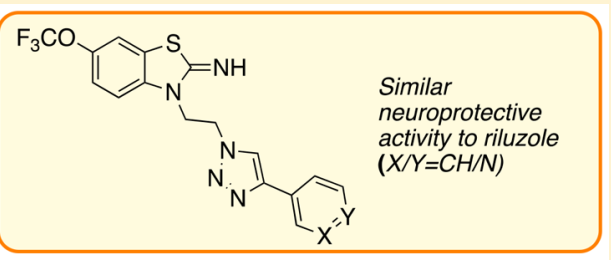

KEYWORDS: Amyotrophic lateral sclerosis, motor neuron disease, riluzole, primary cortical neurons

A myotrophic lateral sclerosis ${ }^{1}$ (ALS, also known as motor neuron disease, or Lou Gehrig's Disease) involves a progressive loss of motor neurons in the central nervous system that control motor functions, usually resulting in death within 3-5 years after diagnosis. Though a rare condition (lifetime incidence ca. 1 in $400^{2}$ ), the disease places enormous social ${ }^{3}$ and financial burdens on both patients ${ }^{4}$ and healthcare programs. The condition is incurable, with riluzole ${ }^{5}$ (1, Rilutek) and edaravone ${ }^{6}$ (2, Radicava) the only drugs currently approved for treatment; both of these drugs provide shortterm palliation, typically extending life by $2-3$ months. In the case of familial ALS (accounting for $5-10 \%$ of cases), there is a genetic mutation that is causative, ${ }^{7}$ but there is currently no consensus on the mode of action of the approved drugs nor agreement on the key cellular targets for new drug-like neuroprotective molecules; thus, there is both scientific and practical value in the design and production of novel chemical matter delivering either mechanistic information or therapeutic activity for ALS. ${ }^{6,8}$ In addition to the two approved drug substances, there is currently a range of small molecule drug candidates $^{9}$ (including peptides, ${ }^{10}$ and saturated ${ }^{11}$ and aromatic $^{12}$ heterocycles) being studied in trials; however, there is still a relative paucity of entirely novel chemical matter for application in ALS therapy.

Riluzole-like heterocyclic small molecule frameworks are also known to demonstrate potential neuroprotective properties; thus, pifithrin- $\alpha(3)^{13}$ contains a partially saturated bicyclic thiazolyl core (rather than the benzothiazolyl scaffold seen in riluzole) and an aromatic substituent placed at the end of a $\mathrm{C}_{2}$ tether attached at the $\mathrm{N}_{1}$ position. A similar substituent pattern is seen in riluzole analogues $\mathbf{4 a}$ and $\mathbf{4 b}$ (Figure 1$)^{5}$ with the
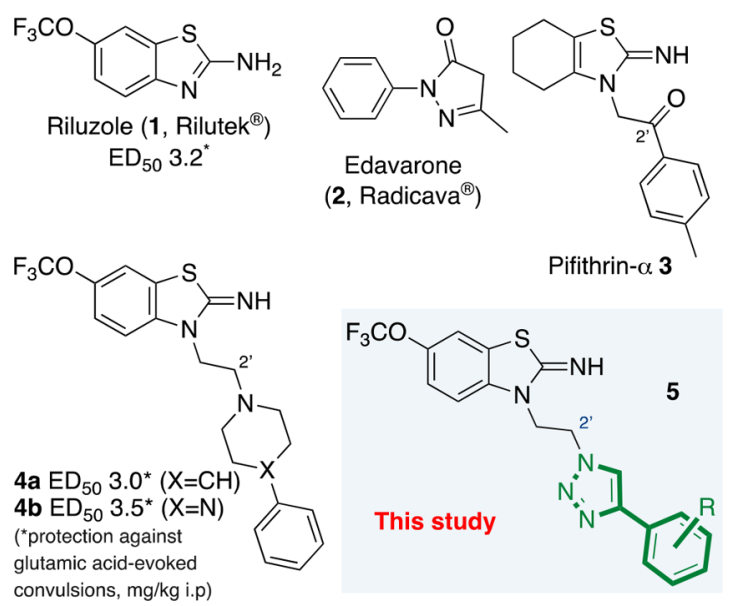

Figure 1. ALS drugs (1 and 2), neuroprotective small molecules (3, $4 a$, and $4 b$ ), and project target (5).

presence of a functionalized ethyl side-chain endowing neuroprotection activity at similar levels to riluzole in rodent models. ${ }^{5}$ As a novel chemical target with potential for neuroprotection and bearing in mind the pyrazinone core of edaravone, we have synthesized previously unreported triazoles 5 as new chemical matter with structural resemblance to riluzole and related molecules.

Special Issue: Med Chem Tech: Driving Drug Development

Received: March 1, 2018

Accepted: May 30, 2018

Published: May 30, 2018 
The synthetic strategy to these compounds revolved around previously unknown lynchpin azide $\mathbf{6}$, which would undergo copper(I)-catalyzed cycloaddition with a range of alkynes to give 5 (Scheme 1). In addition to the triazole functionality

Scheme 1. Click Chemical Strategy for Preparation of Triazole Library 5
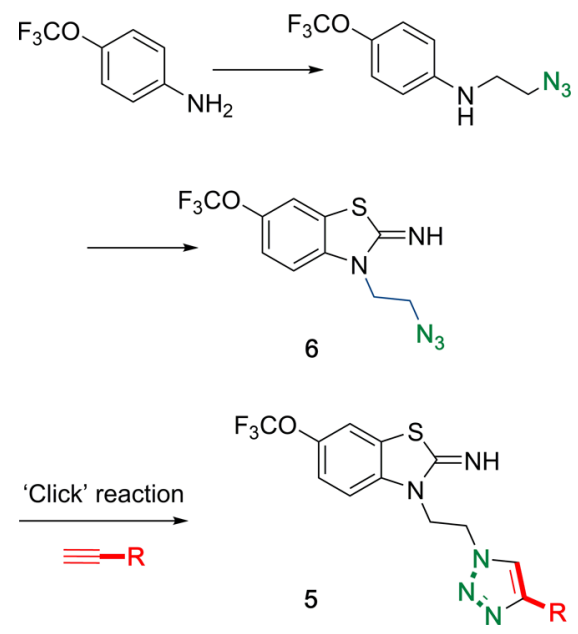

being a known pharmacophoric motif in marketed drug substances (such as tazobactam and cefatrizine), we anticipated that the modularity of the Click process also would allow for incorporation of other functionality (diazirine, dye labels, etc.), thereby facilitating future mechanistic studies.

A major challenge in the design of new therapies for ALS is the fact that the binding site of riluzole is unknown, precluding definitive conclusions to be reached about the mode of action of approved drug substances and novel chemical matter alike. Previous studies using radioligand binding failed to demonstrate any interactions between riluzole and known ligand binding sites on the kainate and NMDA receptors, or the $\mathrm{GABA}_{\mathrm{A}}$ ( $\gamma$-aminobutryic acid) or glycine receptors. ${ }^{14-16}$ In silico docking analysis highlighted the $\mathrm{Na}_{\mathrm{v}} 1.6$ channel. ${ }^{17}$ Since conclusive data to confirm the mechanism of action and binding modes of riluzole remain elusive, limiting the further development of riluzole in the therapeutic pipeline, we envisage tagged chemical probes will facilitate subsequent mechanistic studies once activity has been established.

A complication of the methods used to study the design and development of chemotherapeutics is the fact that the experimental preparations used to determine the mechanism of action of existing and new drugs and drug-like substances vary considerably, with many species and cell type protocols in use. Significantly, many of the cell lines used in the study of ALS are different from the cell types primarily affected in the disease. This presents a challenge in direct comparison of data and makes it difficult both to identify new drugs and to delineate mechanistic rationales. We describe here the synthesis of a novel library of hybrid small molecules containing features of both riluzole and edaravone and the screening of this new chemical matter in novel neuroprotective assays.

Experimental Methods. In order to screen riluzole derivatives, and because of the paucity of robust in vitro assays in this field, it was important to first determine the most suitable cell type, stimulus of cell death, and measure of cell death to measure neuroprotection. Mouse primary cortical neurons and primary motor neurons were cultured using previously described methods. ${ }^{18}$ For screens, neuroprotection assays based on MTT turnover, cell counting, and caspase activation were discarded as riluzole, or derivatives were not protective in these assays of cell death (results not shown). Since dendritic damage and loss is a cardinal feature of the slow degeneration found in motor neuron disease states, we developed morphometric analyses to measure neuronal complexity following disease-relevant stimuli. From a number of challenges tested, the AMPA receptor agonist, kainate was selected to elicit cell damage without rapid cell death. For primary cortical neurons, an antibody against microtubule-associated protein, MAP2, was used in Western blotting experiments and also to stain neuronal dendritic processes. Volocity software was used for perimeter analysis of cell profiles stained with MAP2, and each experimental condition was normalized to the kainate only condition.

Chemical Matter. The target triazole library was accessed in short order on a $100 \mathrm{mg}$ scale according to the synthetic strategy shown in Scheme 1 . Thus, Click reaction of the previously unreported lynchpin azide 6 with a range of alkynes, directly delivered previously unreported triazoles $\mathbf{5 a - 5 a a b}$ (Table 1). Armed with this library of novel chemical matter, we proceeded to the testing program using in vitro model systems.

Results. The library of riluzole derivatives $\mathbf{5 a - 5 a a b}$ was tested on primary cortical neurons for its ability to protect against kainate induced dendritic loss. From this assay, seven compounds were identified as having promise (Figure S2, Supporting Information). Test compounds 5ap, 5ao, 5r, 5g, 5aq, 5w, and 5ak, but not the parent, riluzole (ril), attenuated kainate-induced neurofilament loss. The remaining members of the triazole library did not prevent kainate-induced neurofilament loss (data not shown).

The seven compounds that were positive in the MAP2 assay were also screened in primary cortical neurons, using platebased technology to assay functional properties. In healthy neurons, $\mathrm{K}^{+}$can be used to depolarize neurons and initiate a $\mathrm{Ca}^{2+}$ flux through voltage gated calcium channels. This flux was monitored through incubating cells with fura-2AM for $45 \mathrm{~min}$, then washing and challenging with $10 \mathrm{mM} \mathrm{KCl}$ to induce calcium entry. Neurons pretreated with kainate exhibited a reduced calcium flux, indicating compromised cell function. Riluzole and all the riluzole derivatives were able to attenuate this effect, with $\mathbf{5 a p}$ and $\mathbf{5 g}$ having the greatest effect (results not shown).

We next determined protection using a secondary screen of primary mouse spinal cord motor neurons using the positive compounds from the cortical neuron screen. We first tested a range of outcome assays including cell counting and plate reading of SMI-32 fluorescence, but none provided an output that was both sensitive to disease-relevant stimuli and rescued by riluzole. We therefore employed the antibody SMI- $32^{19}$ to label neurofilaments of motor neurons and Sholl analysis ${ }^{20}$ to monitor neuronal complexity. Following treatments, cells were fixed and stained with SMI-32 antibody, and fluorescent images of cells were captured. Ten individual cells were analyzed for each condition, and the experiment was repeated three times and the data pooled. For Sholl analysis, concentric circles at each $100 \mu \mathrm{m}$ radius from the cell center were drawn, and the number of intersections of processes at each radius interval were measured using image analysis software. The data provided an indication of the arborization and complexity of the motor neuron processes, with reduced process complexity and length preceding cell death. Figure S3 (Supporting Information) shows representative images and analysis from these experiments. Riluzole showed a significant neuroprotective effect, as did two of the test compounds, 5ap and 5ao.

Discussion. Phenyl Derivatives. Phenyl triazole 5a was capable of preventing kainate-induced MAP2 fluorescence loss 
Table 1. Preparation of Triazolyl Library 5a-5aab (Active Compounds Highlighted)

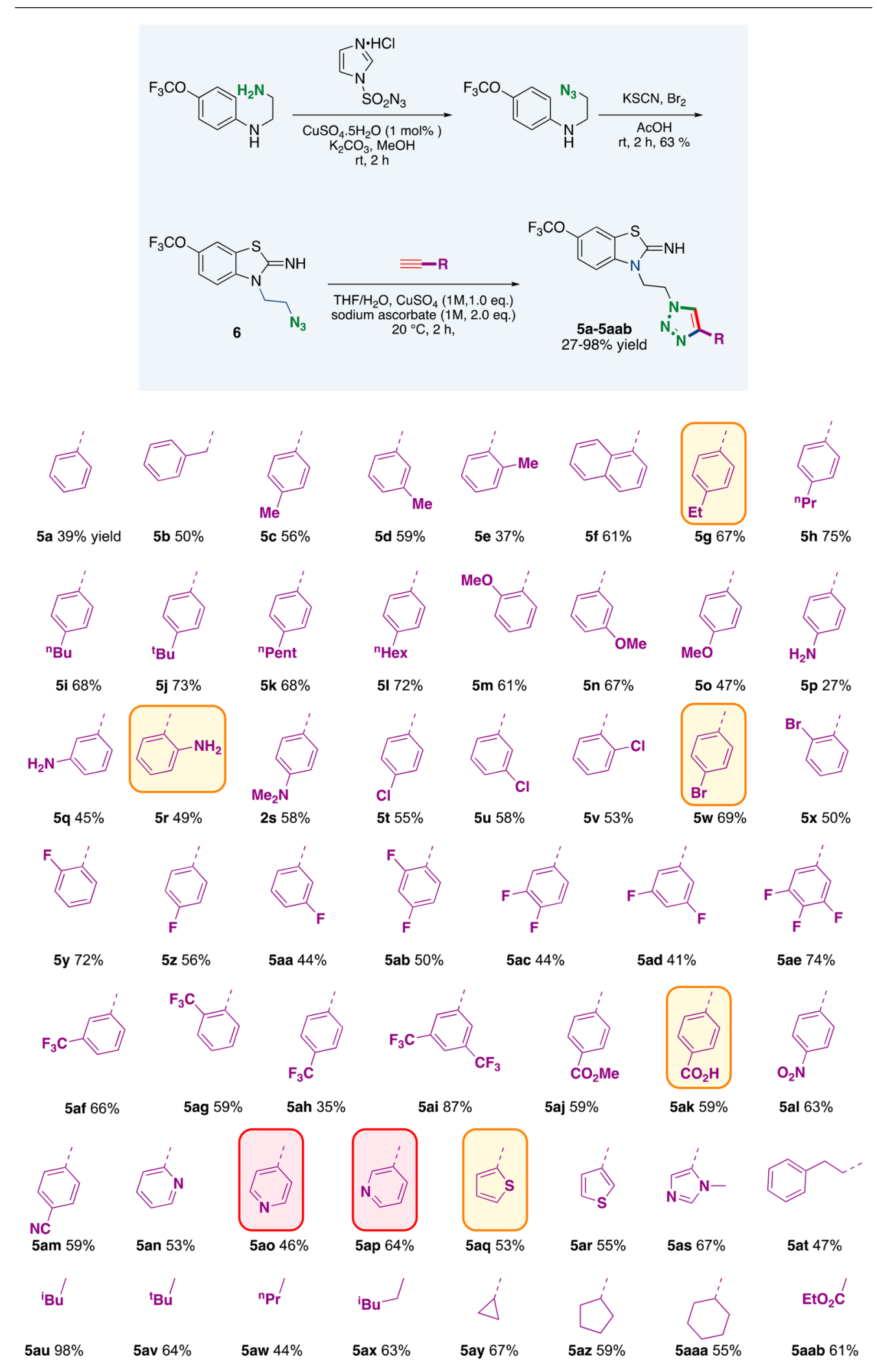

above that of the parent compound, riluzole. However, triazoles where the aryl motif was placed at increasing distances from the heterocycle unit (increasing the length of the carbon chain between the triazole and phenyl ring) were not effective, with compound $\mathbf{5 b}$ (placed at the terminus of a 2 -carbon tether) and 5at (with a 3-carbon tether) showing reduced neuroprotection compared with parent 5a. Compound 5a was not effective in the secondary screen in motor neurons. 
Substituted Phenyl Derivatives. In compounds $5 g-51$, the spatial arrangement of the carbon tether is changed, with the saturated motif placed on the phenyl ring as opposed to the triazolyl unit. Compound $\mathbf{5 g}$, bearing a para- $\mathrm{C}_{2}$-substitutent, showed significant activity against kainate-induced loss in MAP2 in cortical neurons, while compounds $\mathbf{5 h}, \mathbf{5 i}, \mathbf{5 k}$, and $\mathbf{5 1}$ (bearing linear para-carbon chains of 6, 5, 4, and 3 carbons, respectively) did not significantly increase MAP2 fluorescence above that of kainate alone. Compound $\mathbf{5 g}$ attenuated kainite reduction of calcium flux but was not effective in the secondary screen in motor neurons.

Alkyl Triazoles. Deletion of the phenyl ring of the scaffold and replacement with alkyl substituents (5aw, 5ax, and 5au) did not yield compounds with neuroprotective ability as determined by the MAP2 fluorescence assay.

Hetarene Derivatives. Pyridyl-substituted triazoles 5an-5ap included the two most promising compounds; 5ap (with a $3^{\prime}$ substitution pattern) and 5ao ( $4^{\prime}$-substitution): both demonstrated neuroprotection, reduced kainate-induced loss in MAP2, and also prevented motor neuron damage in vitro, with 5ap rescuing kainate-induced reduction in calcium flux in cortical neurons. We note both 5 ao and 5 ap are more active than 2'-pyridyl 5an, which demonstrated increased MAP2 levels but not at a high-enough activity to carry forward into the secondary screen. The $2^{\prime}$-thiophenyl triazole 5 aq was protective in reducing kainite-induced MAP2 loss, but the $3^{\prime}$-isomer, 5ar, had no positive activity.

Electron-Rich Aryl Derivatives. Compound $5 \mathbf{r}$ protected MAP2 fluorescence from kainate treatment and has the addition of an amino group at position 6. Similar derivatives include compounds $\mathbf{5 p}$ and $\mathbf{5 q}$, which have the amino groups at positions 4 and 5, respectively, but neither afforded significant neuroprotection with neuron. Compounds $\mathbf{5 c}$, 5d, and 5 e had the inclusion of a methyl group onto the phenyl ring, at positions 4, 5, and 6, respectively, but none exhibited neuroprotection.

Electron-Deficient Aryl Derivatives. para-(Bromo)phenyl triazole $\mathbf{5 w}$ prevented kainate-induced MAP2 loss in the primary MAP2 screen but was ineffective on motor neurons; its ortho-isomer $5 \mathbf{x}$ had no effect. Chlorination was uniformly unsuccessful: compounds $\mathbf{5 t}-\mathbf{v}$ exhibited no neuroprotection activity. Mono- (compounds 5y-5aa), di- (5ab-5ad), or trifluorinated (5ae) compounds exhibited no neuroprotection activity, while para-(fluoro)phenyl compound $\mathbf{5 z}$ induced increased MAP2 fluorescence but not to an extent great enough to merit further screening. (Trifluoromethyl)phenyl triazoles (5af-5ah) did not exhibit protection. The para(carboxy)phenyl triazole 5ak increased, but the corresponding ester 5aj decreased MAP2 fluorescence compared to kainate alone and demonstrated toxicity in its own right (results not shown).

These data offer the promise of using entirely novel chemical matter for application in ALS therapy, either as drug-like substances or chemical probes. The next phase in this research will focus on structural modification and structure-activity relationship of the small molecules with riluzole-like activity (compounds 5ao and 5ap) and on the use of less active compounds (compounds $\mathbf{5 w}$ and $\mathbf{5 a k}$ ) as start-points for design and implementation of chemical probes (such as "Click" reagents).

Summary. Using a riluzole-triazole hybrid library 5a-5aab and a novel motor neuron screen, we have identified seven new triazoles with neuroprotective activity greater than the parent, riluzole. Of this subset our data shows that the pyridylsubstituted compounds 5ao and 5ap are the most promising, with neuroprotective properties greater than riluzole in two independent in vitro assays on primary neurons. These represent promising chemical start-points for mechanistic studies. We are currently engaged in the design and delivery of chemical probes based on these structures and in the application of this novel chemical matter to further studies of ALS and related conditions.

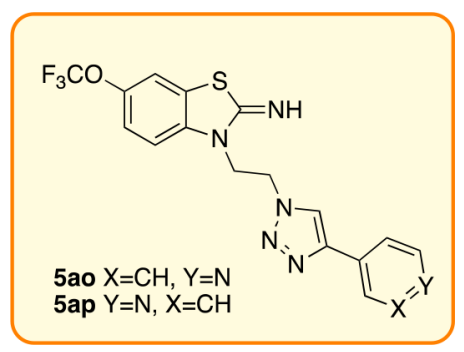

\section{ASSOCIATED CONTENT}

\section{S Supporting Information}

The Supporting Information is available free of charge on the ACS Publications website at DOI: 10.1021/acsmedchemlett.8b00103.

Experimental details for preparation of compounds 5a5aab, including characterization data (PDF)

\section{AUTHOR INFORMATION}

\section{Corresponding Authors}

*E-mail: j.sweeney1@lancaster.ac.uk.

*E-mail: m.rattray@bradford.ac.uk.

ORCID $\odot$

Joseph B. Sweeney: 0000-0001-7593-9598

Notes

The authors declare no competing financial interest.

\section{ACKNOWLEDGMENTS}

We thank the Amyotrophic Lateral Sclerosis Association (ALSA) for project funding, and the Motor Neurone Disease Association, the University of Reading (Ph.D. grant to V.P.), and AstraZeneca (Ph.D. grant to L.A.P.) for other funding.

\section{REFERENCES}

(1) Kiernan, M. C.; Vucic, S.; Cheah, B. C.; Turner, M. R.; Eisen, A.; Hardiman, O.; Burrell, J. R.; Zoing, M. C. Amyotrophic lateral sclerosis. Lancet 2011, 377, 942-55.

(2) Alonso, A.; Logroscino, G.; Jick, S. S.; Hernán, M. A. Eur. J. Neurol 2009, 16, 745-51.

(3) Gibbons, C. J.; Thornton, E. W.; Ealing, J.; Shaw, P. J.; Talbot, K.; Tennant, A.; Young, C. A. Assessing social isolation in motor neurone disease: A Rasch analysis of the MND Social Withdrawal Scale. J. Neurol. Sci. 2013, 334, 112-118.

(4) Vibert, S. MND Costs: Exploring the Financial Impact of Motor Neurone Disease. https://www.demos.co.uk/wp-content/uploads/ 2017/06/DEMJ5449_Financial_impact_report_090617_WEB-6.pdf.

(5) Jimonet, P.; Audiau, F.; Barreau, M.; Blanchard, J. C.; Boireau, A.; Bour, Y.; Coleno, M. A.; Doble, A.; Doerflinger, G.; Huu, C. D.; Donat, M. H.; Duchesne, J. M.; Ganil, P.; Gueremy, C.; Honor, E.; Just, B.; Kerphirique, R.; Gontier, S.; Hubert, P.; Laduron, P. M.; Le Blevec, J.; Meunier, M.; Miquet, J. M.; Nemecek, C.; Pasquet, M.; Piot, O.; Pratt, J.; Rataud, J.; Reibaud, M.; Stutzmann, J.-M.; Mignani, S. Riluzole series. Synthesis and in vivo "antiglutamate" activity of 6- 
substituted-2-benzothiazolamines and 3-substituted-2- imino-benzothiazolines. J. Med. Chem. 1999, 42, 2828-2843.

(6) Kiernan, M. C. Motor neuron disease in 2017: Progress towards therapy in motor neuron disease. Nat. Rev. Neurol. 2018, 14, 65-66.

(7) Rosen, D. R.; Siddique, T.; Patterson, D.; Figlewicz, D. A.; Sapp, P.; Hentati, A.; Donaldson, D.; Goto, J.; O’Regan, J. P.; Deng, H.-X.; Rahmani, Z.; Krizus, A.; McKenna-Yasek, D.; Cayabyab, A.; Gaston, S. M.; Berger, R.; Tanzi, R. E.; Halperin, J. J.; Herzfeldt, B.; Van den Bergh, R.; Hung, W.-Y.; Bird, T.; Deng, G.; Mulder, D. W.; Smyth, C.; Laing, N. G.; Soriano, E.; Pericak-Vance, M. A.; Haines, J.; Rouleau, G. A.; Gusella, J. S.; Horvitz, H. R.; Brown, R. H., Jr Mutations in Cu/Zn superoxide dismutase gene are associated with familial amyotrophic lateral sclerosis. Nature 1993, 362, 59-62.

(8) Özdinler, P. H.; Silverman, R. B. Treatment of Amyotrophic Lateral Sclerosis: Lessons Learned from Many Failures. ACS Med. Chem. Lett. 2014, 5, 1179-1181.

(9) Drugs in Development Database. http://www.alsresearchforum. org/research-tools/drugs-in-development-database.

(10) Wu, B.; De, S. K.; Kulinich, A.; Salem, A. F.; Koeppen, J.; Wang, R.; Barile, E.; Wang, S.; Zhang, D.; Ethell, I.; Pellecchia, M. Potent and Selective EphA4 Agonists for the Treatment of ALS. Cell Chem. Biol. 2017, 24, 293-305.

(11) Mavlyutov, T. A.; Guo, L. W.; Epstein, M. L.; Ruoho, A. E. Role of the Sigma-1 receptor in Amyotrophic Lateral Sclerosis (ALS). J. Pharmacol. Sci. 2015, 127, 10-6.

(12) See, for instance Benatar, M.; Wuu, J.; Andersen, P. M.; Atassi, N.; David, W.; Cudkowicz, M.; Schoenfeld, D. Randomized, doubleblind, placebo-controlled trial of arimoclomol in rapidly progressive SOD1 ALS. Neurology 2018, 90, e565-e574.

(13) Zhu, X.; Yu, Q.-s.; Cutler, R. G.; Culmsee, C. W.; Holloway, H. W.; Lahiri, D. K.; Mattson, M. P.; Greig, N. H. Novel p53 Inactivators with Neuroprotective Action: Syntheses and Pharmacological Evaluation of 2-Imino-2,3,4,5,6,7-hexahydrobenzothiazole and 2Imino-2,3,4,5,6,7-hexahydrobenzoxazole Derivatives. J. Med. Chem. 2002, 45, 5090-5097.

(14) Benavides, J.; Camelin, J. C.; Mitrani, N.; Flamand, F.; Uzan, A.; Legrand, J. J.; Gueremy, C.; Le Fur, G. 2-Amino-6-trifluoromethoxy benzothiazole, a possible antagonist of excitatory amino acid neurotransmission-II. Biochemical properties. Neuropharmacology 1985, 24, 1085-1092. Debono, M. W.; Le Guern, J.; Canton, T.; Doble, A.; Pradier, L. Inhibition by riluzole of electrophysiological responses mediated by rat kainate and NMDA receptors expressed in Xenopus oocytes. Eur. J. Pharmacol. 1993, 235, 283-289. He, Y.; Benz, A.; Fu, T.; Wang, M.; Covey, D. F.; Zorumski, C. F.; Mennerick, S. Neuroprotective agent riluzole potentiates postsynaptic GABA(A) receptor function. Neuropharmacology 2002, 42, 199-209.

(15) Debono, M. W.; Le Guern, J.; Canton, T.; Doble, A.; Pradier, L. Inhibition by riluzole of electrophysiological responses mediated by rat kainate and NMDA receptors expressed in Xenopus oocytes. Eur. J. Pharmacol. 1993, 235, 283-289.

(16) He, Y.; Benz, A.; Fu, T.; Wang, M.; Covey, D. F.; Zorumski, C. F.; Mennerick, S. Neuroprotective agent riluzole potentiates postsynaptic GABA(A) receptor function. Neuropharmacology 2002, 42, 199-209.

(17) Sierra Bello, O.; Gonzalez, J.; Capani, F.; Barreto, G. E. In silico docking reveals possible Riluzole binding sites on Nav1.6 sodium channel: implications for amyotrophic lateral sclerosis therapy. $J$. Theor. Biol. 2012, 315, 53-63.

(18) Rainey-Smith, S. R.; Andersson, D. A.; Williams, R. J.; Rattray, $M$. Tumour necrosis factor alpha induces rapid reduction in AMPA receptor-mediated calcium entry in motor neurones by increasing cell surface expression of the GluR2 subunit: relevance to neurodegeneration. J. Neurochem. 2010, 113, 692-703.

(19) Tsang, Y. M.; Chiong, F.; Kuznetsov, D.; Kasarskis, E.; Geula, C. Motor neurons are rich in non-phosphorylated neurofilaments: crossspecies comparison and alterations in ALS. Brain Res. 2000, 861, 4558.

(20) Sholl, D. A. Dendritic organization in the neurons of the visual and motor cortices of the cat. J. Anat. 1953, 87, 387-406. 